\title{
Analysis of CT and MRI Combined Examination for the Diagnosis of Acute Cerebral Infarction
}

\author{
Wenbo Zhang, Jingliang Cheng, Yong Zhang, Keyan Wang and Hongrui Jin \\ Department of Magnetic Resonance, The First Affiliated Hospital of Zhengzhou University, Zhengzhou, China
}

\begin{abstract}
The aim of this study was to analyse the application value of CT and MRI combined examination for the diagnosis of acute cerebral infarction. It was an observational study carried out from March 2017 to April 2018. All the patients of acute cerebral infarction, diagnosed on the basis of clinical and laboratory findings and underwent brain CT and MRI examination within 6 hours after the onset of the disease, were included in the study. A total of 70 patients were diagnosed, which included $40(57.14 \%)$ males and $30(42.86 \%)$ females. Median age was 64 years. The confirmed diagnosis rate of acute cerebral infarction by CT combined with MRI was higher than that by CT examination alone $(p<0.001)$. The positive rates of the brainstem were statistically different between the two methods $(p=0.007)$. The diameter of infarct lesion in CT combined with MRI was longer than that in CT examination alone $(p<0.001)$. CT combined with MRI can be used as the first choice for early diagnosis of acute cerebral infarction, particularly of brain stem.
\end{abstract}

Key Words: Acute cerebral infarction, Early diagnosis, Lesion, CT, MRI.

How to cite this article: Zhang W, Cheng J, Zhang Y, Wang K, Jin H. Analysis of CT and MRI combined examination for the diagnosis of acute cerebral infarction. J Coll Physicians Surg Pak 2019; 29(9):898-9.

Cerebral infarction, also called cerebral arterial thrombosis, refers to the focal ischemic necrosis in brain tissue or cerebromalacia caused by a disturbance of cerebral blood circulation. 1 Acute cerebral infarction mostly occurs in elderly people. The curative effect of thrombolytic therapy was the best within 1 to 6 hours after onset for patients with acute cerebral infarction. Missing this time window means losing the chance of best thrombolytic effect. Clinical early diagnosis of acute cerebral infarction is the key to ensure the effect, as well as the prerequisite for the implementation of thrombolytic therapy. Therefore, active and effective early diagnosis is of great clinical significance for patients with acute cerebral infarction. CT and MRI are often used to diagnose the disease clinically. CT diagnosis is fast with high sensitivity to acute cerebral hemorrhage. Conventional sequences such as T1W1 in MRI can eliminate the interference caused by bone artifacts in the lesion. ${ }^{2}$

The aim of this study was to discuss the value of CT and $\mathrm{MRI}$ in the joint examination of acute cerebral infarction, in order to provide a reference for early diagnosis and treatment of acute cerebral infarction.

This observational study was carried out at the First Affiliated Hospital of Zhengzhou University, China, from March 2017 to April 2018. The study was conducted

Correspondence to: Jingliang Cheng, Department of Magnetic Resonance, The First Affiliated Hospital of Zhengzhou University, Zhengzhou, 450052, China

E-mail:wmfoh3@163.com

Received: June 25, 2018; Revised: January 23, 2019;

Accepted: March 26, 2019 after approval from the Hospital Ethical Committee. Patients clinically diagnosed as acute cerebral infarction, who underwent CT and MRI examination within 6 hours of onset, were included. Patients combined with cancer, hematological diseases, etc. and unable to undergo CT or MRI examination, were excluded.

The patients were examined by CT after admission, and then MRI was performed. 128-slice spiral CT produced by Siemens was used to perform continuous scans on the patient; the scan range was the entire brain; the scan parameters were axial scans or spiral scans; the voltage was $120 \mathrm{KV}$, current $150 \mathrm{~mA}$, layer thickness $5.0 \mathrm{~mm}$ and layer distance $5.0 \mathrm{~mm}$. GE 1.5T MRI production was used to perform whole brain scan on the patient. Parameters were selected as field of view (FOV) $230 \mathrm{~mm}$, layer thickness $6 \mathrm{~mm}$, layer distance $1.5 \mathrm{~mm}$ for multiparameter head axial scanning. The overall detection rate of acute cerebral infarction and the detection rate of different infarct parts by CT alone and CT combined with MRI were observed. The mean maximum diameter of acute cerebral infarction was compared between the two examination methods. Collected data were analysed using paired t-test and Chi-square test by Statistical Package for Social Sciences (SPSS) version 19. P-value of less than 0.05 was considered significant.

A total of 70 patients included $40(57.14 \%)$ males and 30 $(42.86 \%)$ females. Median age was 64 years. Among the 70 patients, 51 cases $(72.86 \%)$ were diagnosed by CT examination alone, and 68 cases $(97.14 \%)$ were diagnosed by CT combined with MRI, the difference was statistically significant $(p<0.001)$. There was no 
Table I: Comparison of diagnosis results of different examination methods.

\begin{tabular}{l|c|c|c|c|c|c|c|c}
\hline Check method & $\mathrm{n}$ & Frontal lobe & Temporal lobe & Basal ganglia & Occipital lobe & Cerebellum & Brain stem & Confirmed cases \\
\hline CT & 70 & $8(11.43)$ & $13(18.57)$ & $17(24.29)$ & $9(12.86)$ & $4(5.71)$ & $0(0)$ & $51(72.86)$ \\
CT and MRI & 70 & $10(14.29)$ & $14(20.00)$ & $21(30.00)$ & $10(14.29)$ & $6(8.57)$ & $7(10.00)$ & $68(97.14)$ \\
p-value & & 0.614 & 0.830 & 0.447 & 0.805 & 0.512 & 0.007 & $<0.001$ \\
\hline
\end{tabular}

significant difference in the positive rates for detection of frontal lobe, temporal lobe, basal ganglia, occipital lobe and cerebellum between the two examination methods ( $p=0.614,0.830,0.447,0.805$, and 0.512 , respectively). However, CT combined with MRI could detect brain stem infarct while CT alone could not. The difference in the positive rate of brainstem was statistically significant $(p=0.007)$, as shown in Table I. The mean maximum diameter of lesions on CT and CT combined with MRI was $7.63 \pm 1.08 \mathrm{~cm}$ and $9.27 \pm 1.91 \mathrm{~cm}$, respectively; and there was a significant difference between the two methods $(p<0.001)$.

Patients with acute cerebral infarction usually undergo CT examination after admission. The main reason is that CT examination has the characteristics of shorter time, low price, etc., and the contraindications are relatively fewer than MRI. Timely CT examinations on patients can also exclude cases of cerebral hemorrhage. In this study, the diagnostic accuracy of $72.86 \%$ (51 cases) was obtained after CT examination in 70 patients, which was relatively low. The cause of this problem is that in the early stage, patients do not have a relatively significant degree of edema, so the mass effect is not obvious. ${ }^{3}$ The diagnosis rate of the patients with no significant edema is low. The diagnostic accuracy rate of $97.14 \%$ (68 cases) was obtained in 70 patients after combining MRI examination, which was more accurate than that of CT alone. The results of this study were consistent with those of Amorim et al. ${ }^{4}$ The reason may be that MRI is mainly based on the changes in the water content in the brain tissue. It can detect the early edema of cell toxicity in patients within six hours or less after onset; the water content of local infarct lesion increases, which helps prolong the nuclear magnetic signal and improve the diagnosis rate. This study also showed that the diameter of infarct lesion in patients with CT combined with MRI was larger than that of CT examination alone. It was mainly because the early lesions of acute cerebral infarction mainly consisted of infarct foci and the surrounding penumbra. MRI examination showed clearer penumbra, so the infarct lesion diameter was larger. ${ }^{5}$ Because there was no bone artifact in MRI, it was possible to detect small infarct foci such as brainstem when CT was combined with MRI, while the brain stem infarction could not be detected by CT alone.

This study shows that diagnosis rate was higher using CT combined with MRI, particularly for brain stem infarction; and the determination of the lesion was more accurate, which can be used as the first choice for early diagnosis of acute cerebral infarction.

\section{ETHICAL APPROVAL:}

The study was conducted after approval from the Hospital Ethical Committee.

\section{CONFLICT OF INTEREST:}

Authors declared no conflict of interest.

\section{AUTHORS' CONTRIBUTION:}

WZ: Drafted the work; analysed and interpretated data for the work.

JC: Drafted the work and finally approved to be published. $\mathrm{YZ}, \mathrm{KW}$ : Drafting the work and revised critically.

$\mathrm{HJ}$ : Contributed to the conception/design of the work and revised it critically.

\section{REFERENCES}

1. Kobayashi $\mathrm{Y}$, Takamatsu R, Watanabe R, Sato S. Cerebral infarction with leriche syndrome. Intern Med 2018; 57:1953-4.

2. Saberi A, Roudbary SA, Ghayeghran A, Kazemi S, Hosseininezhad M. Diagnosis of meningitis caused by pathogenic microorganisms using magnetic resonance imaging: A systematic review. Basic Clin Neurosci 2018; 9: 73-86.

3. Dharmasaroja PA. Fluid intake related to brain edema in acute middle cerebral artery infarction. Trans/ Stroke Res 2016; 7: 49-53.

4. Amorim RL, de Andrade AF, Gattás GS, Paiva WS, Menezes M, Teixeira MJ, et al. Improved hemodynamic parameters in middle cerebral artery infarction after decompressive craniectomy. Stroke 2014; 45:1375-80.

5. Yamaguchi S, Horie N, Morikawa M, Tateishi Y, Hiu T, Morofuji Y, et al. Assessment of veins in T2*-weighted MR angiography predicts infarct growth in hyperacute ischemic stroke. Plos One 2018; 13:e0195554. 